\title{
Delivery Probability Comparison of VDTN Routing Protocols
}

Megha Bhatt ${ }^{1}$ and Rima Shelat ${ }^{2}$

\begin{abstract}
Delay tolerant network (DTN) has been utilized for intermittent network, where no of hosts are movable, there is no fixed topology and connection is opportunistic. It is challenging to transmit data in such opportunistic environment. DTN is also implemented in Vehicular known as Vehicular delay tolerant network (VDTN). The message transmission in VDTN using different routing protocols needs to be improved as VDTN suffers from the high speed of vehicles, short contact time and unstable topology and very high delay for message passing. The objective of this paper is to do comparative analysis of five different protocols of VDTN with variance of various parameters and suggest one protocol for optimum performance concentrating delivery probability. Delivery probability is compared on parameters - variation in no of nodes, buffer size and transmission data rate. Rating based system is used to analyze comparative study. All protocols are implemented using The ONE simulator.

Keywords: DTN, VDTN, Epidemic Routing, Direct Delivery Routing, Spray and Wait Routing, Binary Spray and Wait Routing, Prophet Routing, Delivery Probability, Buffer Size, Transmission Speed, Number of Nodes
\end{abstract}

\section{Introduction}

Delay Tolerant Network (DTN) is Mobile Network where nodes are movable and also known as an intermittently connected network. When your modem/router drops connection at random intervals is known as an intermittent connection. Delay tolerant network is wireless network where there is no end to end path available or the probability of an end to end path from source to destination is very low. In DTN all the nodes are movable so connectivity for transmission from source to destination is only possible when nodes come into transmission range of each other. Transmission mode can be any wireless media. (Infrared, Bluetooth, Wi-Fi, WI-Max). Nodes are continuously movable so in the DTN end to end transmission may not possible so DTN avails hop to hop transmission. If one node has a message to deliver, but not connected to destination, so it sends messages to first connected node, but if any other node is not in transmission range of the sender, it stores the message till the time an appropriate link is not established between the sender and another node. A communication opportunity between two nodes is called a contact in DTN's. [1]

DTN has different contact available as it is a large group of network. They are as below. [1]

- $\quad$ Persistent contact: Persistent contact means the contact is always available

\footnotetext{
${ }^{1}$ Bholabhai Patel College Of Computer Studies (BCA), Gandhinagar, Gujarat, India

${ }^{2}$ Bholabhai Patel College Of Computer Studies (BCA), Gandhinagar, Gujarat, India
} 
- $\quad$ On-demand contact: On-demand contact means a contact can be initiated when needed

- Scheduled contact: Scheduled contact means the contact and its characteristics is known in advance

- Opportunistic contact: Opportunistic contact means when nodes get the opportunity to connect, then only connections established. There is no guarantee of connection. It is called opportunistic DTN.

Protocols for Mobile ad hoc network (MANET) is designed with the idea that the network is fully connected and path between nodes exist always or if paths are down then they are only for a very short period of time. Routing protocols of MANET transmit messages only if full path is established between sender and receiver, if there is no path established protocol is failed to transmit data, but it is not possible for DTN because it is having intermittent connection and there is no surety to the establishment direct path between source and destination. So MANET protocols will not work for DTN. To overcome routing problems DTN provides Store - Carry and Forward (SCF) functionality. DTN nodes have buffers to store messages. DTN routing protocols use nodes movements and message buffering for transmission of messages from source to destination. [1]

Store and forward message switching is used to successfully transfer message with intermittent connection, long delay, variable data rate and high error rates. Actually, this is a very old method used in the postal system in ancient time, where whole message or the part (packets) of the message will be transferred to one node and then another and so on in the way of destination node and gradually it reaches to the destination.

Routers of DTN networks want persistent storage for their queues for below reasons: [2]

- A communication link to the next node, possibly not available for a long period of time

- $\quad$ There are chances to message may be transmitted again if there is an error while transmitting messages or if a contacted node doesn't accept of a forwarded message

- When whole message is transferred, the message switching method provides the size of message with it so at that time there is a need of immediate storage space and bandwidth and also retransmission needed in some cases.

\section{Problem definition and Scope}

\section{Problem definition:}

Since the nature of DTN is like opportunistic network connection, where there is no surety that message will reach to the destination with a certain delay. Many routing protocols are developed to meet the challenges of the opportunistic network to successful message transmission. Research on DTN and VDTN routing protocols is still in its infancy, it is unclear which protocol can be used in specific situations with the variance of different parameters to get optimum results in terms of Delivery Probability.

\section{Objective and Scope:}

The objective is to compare performance of five routing protocols - Epidemic routing, Direct Delivery routing, Spray and Wait routing, Binary Spray and Wait routing and PRoPHET routing for VDTN with variance of three input metrics - number of nodes, buffer size and transmission speed. The Elaborative comparative and analytical study of routing protocols in VDTN may be useful to the network administrator who wants to implement protocols in real scenario to achieve optimum result. Through the study researcher can go for further research in the domain of VDTN protocols for the improvement of the protocol and to achieve more improved performance of the network. 


\section{Routing Protocols}

\section{Direct Delivery Routing:}

In direct delivery routing (DDR) does end to end message transmission. In DDR Source node stores message till the time it comes into direct contact with the destination node. Message delivery probability using DDR is extremely low as it needs end to end path between source and destination that too in such intermittent network. If source and destination contact is not established, then the message would not be delivered. [3]

\section{Epidemic Routing:}

In Epidemic routing protocol the node which receives message will send a copy of message to nodes come into its contact. Each node who is having message will send the copy of it to encounter nodes such as the message will spread to all the nodes of the network. The epidemic routing algorithm may be the optimum efforts to reach message to the destination node. Each node maintains summary vector in its buffer which contains the actual message with its unique identifier. When any node comes to the transmission range of another node they compare summary vectors and transmit messages which are not available in current summary vector. Moreover, it is possible to sidestep to compare summary vector again and again with the same node when they encounter frequently, the history of recent contact is maintained in the node's buffer. Epidemic routing obtains the optimal path to a destination with the smallest delay if there are enough buffer size and communication bandwidth between nodes. Epidemic routing searches all possible path for message transmission to transmit messages and so that node failure doesn't harm. The main drawback of Epidemic routing is - It works on forwarding multiple copies of the same message so that there may be wastage of buffer, bandwidth and nodes energy. When resources are limited, there are high chances of message dropping. [3], [4]

\section{Spray and Wait routing:}

In spray and wait there is restriction on making copies of the message. Only L number of copies are allowed which, depending on the number of available nodes in the network. There are two main stages of spray and wait routing protocol. 1) The spray phase and 2) The wait phase. One number is attached when message is created which shows maximum possible copies of the message. The source node is spread messages to different nodes. When a node receives a copy of the message it enters to wait phase. Nodes hold message till the time it directly meet to the next node/destination.

\section{Normal Spray and Wait}

In vanilla version, the source node creates $\mathrm{L}$ no. of copies and it distributes one copy of that message to total L-1 nodes which first encounter it. Then each node goes to wait phase till the time it comes to the direct contact of destination.

Binary Spray and Wait In binary spray and wait source also makes the L copies of the created message. It transmits L/2 of those copies to first node it intersects, and is again copying messages and gives to encounters nodes, then after each encountered node transmit half no of total received copies to another encountered node, but it cannot copy messages. This process goes on till the time nodes are having single copy available. At this stage they go to wait phase. Each node which is having message will wait for direct transmission opportunities with the destination node.

\section{ProPhet routing (Probabilistic Routing Protocol uses History of Encounters and Transitivity)}

In PROPHET routing protocol, it is presumed that all the mobile nodes have some pattern to move into network. All nodes have information about another nodes' probable movement on the bases of past encountered nodes. Because of this history nodes may have knowledge that which nodes are frequently or recently encountered. So it would be easy to determine prediction. Thus, in PROPHET movements of nodes is not totally unplanned but deterministic characteristics. PROPHET creates 
one probabilistic metric which is known as delivery predictability $\mathrm{P}(\mathrm{A}, \mathrm{B})$. This metric check the probability of node A will be able to transmit messages to destination node $\mathrm{B}$. When one node encounters another node, it will pass summary vector to that node and this process will go on until message reach to the destination. It seems similar to Epidemic routing, but the only difference between Epidemic routing and PROPHET routing is a Prophet summary vector also contains delivery predictability value to reach to the destination node. When a message is being delivered to intermediate node, it will just share the delivery probability. Intermediate node will not update that probability to make out which node has highest delivery probability to the destination node. PROPHET method is having two phases. In the first phase it will calculate the delivery probability to send messages to the destination and in phase two it uses forwarding strategies.

\section{Parameter explanation}

- $\quad$ Transmission speed: The data rate at which message is sent and received across two or more nodes over a communications channel. (Kbps)

- $\quad$ Buffer size: Size of the message buffer. (Bytes)

- $\quad$ Number of nodes: Number of hosts in the group.

- $\quad$ Delivery probability: Probability that message can be received from the destination (between 0 to 1 ) delivery capacity of the routing protocol.

Table 1: Simulation Setup Information

\begin{tabular}{|l|l|}
\hline PARAMETERS & VALUE \\
\hline Simulation Time & $10000(\mathrm{Sec})$ \\
\hline Interface & Bluetooth Interface \\
\hline Interface type & SimpleBroadcastInterface \\
\hline Transmission range & $10($ meter $)$ \\
\hline Transmission speed & $100,200,300,400 \mathrm{k}, 500(\mathrm{kbps})$ \\
\hline Buffer size & $1,2,5,10,20(\mathrm{MB})$ \\
\hline No. of nodes & $50,100,150,200,250$ \\
\hline Message size & $500 \mathrm{~KB}$ to $1 \mathrm{MB}$ \\
\hline Message TTL & 300 minutes \\
\hline
\end{tabular}

A. Delivery probability with various buffer size.

V. Experimental Result Analysis 


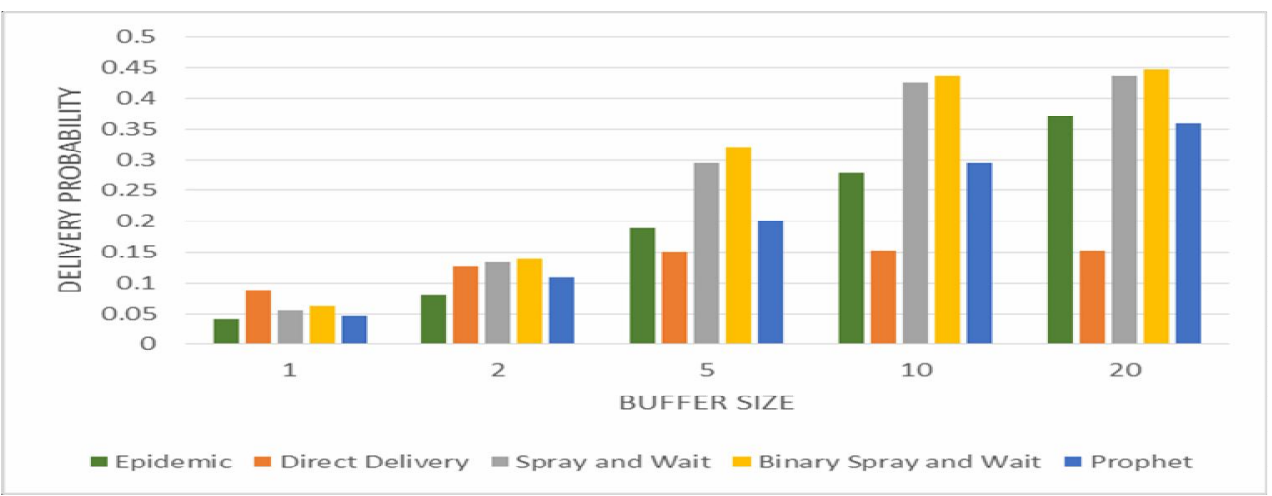

Figure 1. Comparison chart buffer size vs delivery probability

Figure 1 clearly indicates two variables. Response variable - delivery probability and explanatory variable - buffer size. The comparison chart of the delivery probability for Epidemic routing, Direct Delivery Routing, Spray and Wait Routing, Binary Spray and Wait Routing and Prophet Routing shows that with the increment of buffer size, delivery probability is also increased of all four protocols except Direct Delivery Routing. Direct Delivery routing is having a minor increment compare to others. Because the Direct Delivery routing uses hand-to-hand packet delivery strategy. Binary Spray and Wait gives excellent performance for delivery probability in comparison of other protocols. (Transmission Speed is $250 \mathrm{k}$ and Number of nodes are 40)

B. Delivery probability with various Transmission Speed

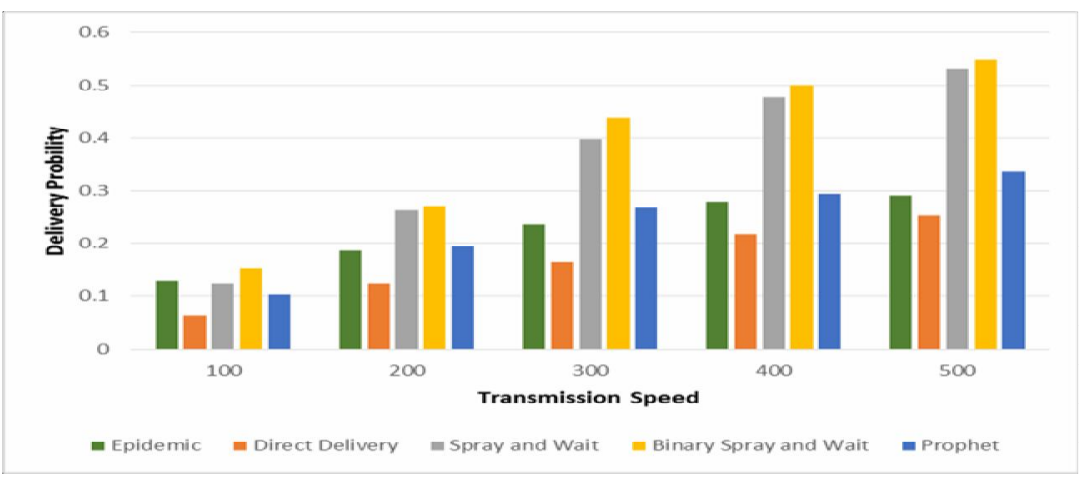

Figure 2. Comparison chart Transmission speed vs delivery probability 
Figure 2 clearly indicates two variables. Response variable - delivery probability and explanatory variable - Transmission Speed. The comparison chart of the delivery probability for Epidemic routing, Direct Delivery Routing, Spray and Wait Routing, Binary Spray and Wait Routing and Prophet Routing shows that with the increment of Transmission speed, delivery probability is also increased. Binary Spray and Wait gives excellent performance for delivery probability in comparison of other protocols. While Direct Delivery gives lowest performance compared to all routing protocols. Binary Spray and Wait as well as Spray and wait provides optimum probability due to its nature of creating copies and passing those copies to other encountered nodes, which increase the probability of moving the message to the destination while Direct Delivery works on predefined end to end path which adversely effect on the delivery probability in such intermittent network environment. (The buffer size is $5 \mathrm{MB}$ and Number of nodes are 40)

C. Packet delivery probability with various Number of Nodes

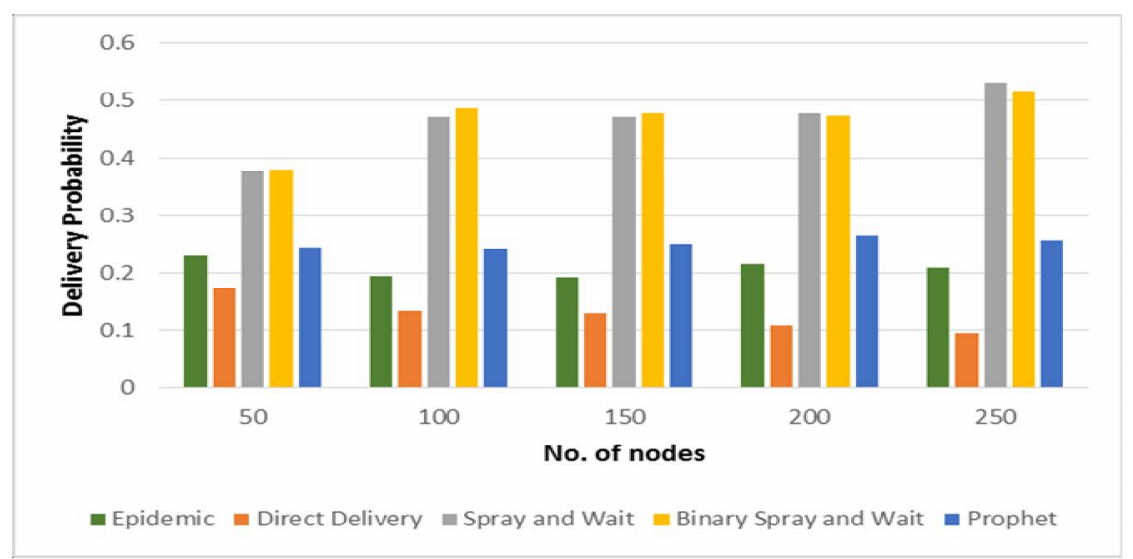

Figure 3. Comparison chart number of nodes vs delivery probability

Figure 3 clearly indicates two variables. Response variable - delivery probability and explanatory variable - Number of Nodes. The comparison chart of the delivery probability for Epidemic routing, Direct Delivery Routing, Spray and Wait Routing, Binary Spray and Wait Routing and Prophet Routing shows that with the increment of Number of nodes, delivery probability is also increased of Spray and Wait Routing as well as Binary Spray and Wait Routing. While it is clear from the chart that Epidemic Routing, Direct Delivery Routing and Prophet Routing is not capable to increase the delivery probability with the increment of nodes. (Transmission Speed is $250 \mathrm{k}$ and Buffer size is $5 \mathrm{MB}$ )

For exhaustive comparative study rating method is applied on the simulated value based results plotted in the above charts. Rating system works as mentioned in Table 2.

Table: 2 Rating Method while concentrating on message delivery probability is as follows.

\begin{tabular}{|l|l|l|}
\hline SR. NU & Readings & Rating \\
\hline 1 & For highest delivery probability & 5 \\
\hline 2 & For second highest delivery probability & 4 \\
\hline 3 & For third highest delivery probability & 3 \\
\hline
\end{tabular}




\begin{tabular}{|l|l|l|}
\hline 4 & For forth highest delivery probability & 2 \\
5 & For lowest delivery probability & 1 \\
\hline
\end{tabular}

According to above rating criteria, rating is assigned to Delivery probability of all routing protocols with the variance of all three metrics to evaluate performance of routing protocols.

Table: 3 Rating concentrating message delivery probability with total and mean

\begin{tabular}{|c|c|c|c|c|c|}
\hline Protocols-- $\square$ & Epidemic & Direct & Spray and WaitBinary & Spray & and Prophet \\
\hline Buffer size 1m & 1 & 5 & 3 & & 2 \\
\hline Buffer size $2 \mathrm{~m}$ & 1 & 3 & 4 & & 2 \\
\hline Buffer size 5m & 2 & 1 & 4 & & 3 \\
\hline Buffer size $10 \mathrm{~m}$ & 2 & 1 & 4 & & 3 \\
\hline Buffer size $20 \mathrm{~m}$ & 3 & 1 & 4 & & 2 \\
\hline Trans. Speed100 & 4 & 1 & 3 & & 2 \\
\hline Trans. Speed200 & 2 & 1 & 4 & & 3 \\
\hline Trans. Speed300 & 2 & 1 & 4 & & 3 \\
\hline Trans. Speed400 & 2 & 1 & 4 & & 3 \\
\hline Trans. Speed500 & 2 & 1 & 4 & & 3 \\
\hline No of nodes 50 & 2 & 1 & 4 & & 3 \\
\hline No of nodes100 & 2 & 1 & 4 & & 3 \\
\hline No of nodes 150 & 2 & 1 & 4 & & 3 \\
\hline No of nodes 200 & 2 & 1 & 5 & & 3 \\
\hline No of nodes 250 & 2 & 1 & 5 & & 3 \\
\hline Total & 31 & 21 & 60 & & 41 \\
\hline Mean & 2.066666667 & 1.4 & 4.8 & & 2.733333333 \\
\hline
\end{tabular}

Now it is clear that Binary Spray and Wait gives optimum delivery probability based on Table 3. Same result is graphically represented in figure 3.

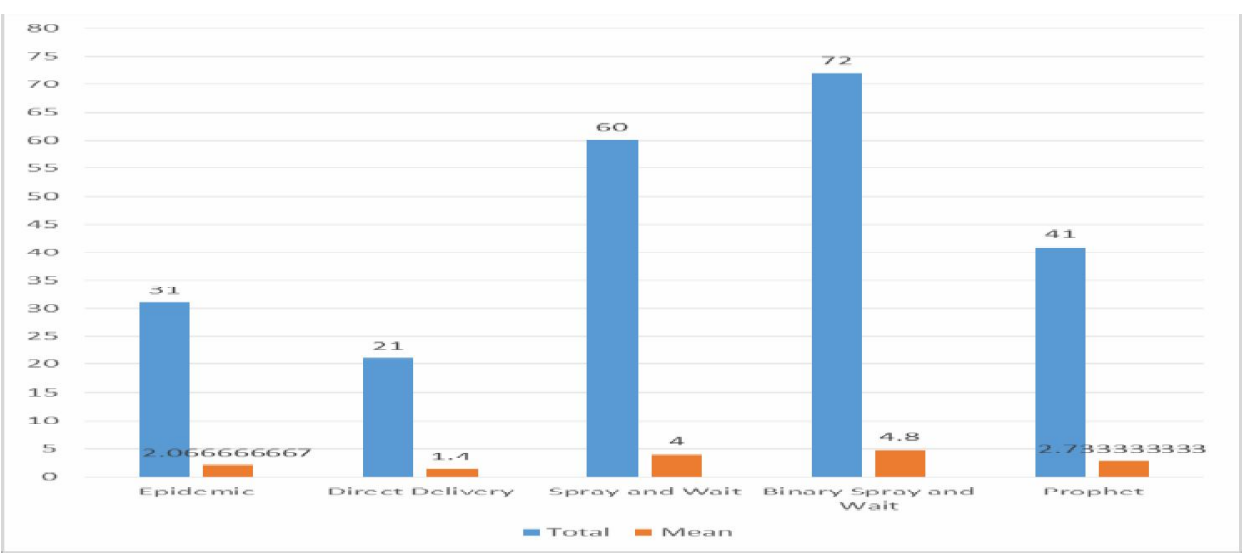

Figure 4. Comparison chart Total and mean of all three parameters value 


\section{Conclusion and Future scope}

Delivery probability comparison of all protocols using the variance of buffer size, transmission speed and number of nodes it is clear that Binary Spray and Wait gives excellent performance in all scenarios shown in Table 3 and Figure 4. Second optimum performance is of Spray and Wait Routing, Prophet Routing gives third optimum result, forth optimum result is of Epidemic Routing and Direct Delivery Routing provides lowest delivery probability compare to other routing protocols. The in depth comparison may useful to network administrator to install protocols in real scenario to gain optimum network performance.

In future researcher can derive rating based comparison of other parameters of VDTN to evaluate performance. Through such exhaustive comparison researcher may know which protocol should be improved to improve overall performance of the network.

\section{REFERENCES}

[1] Chintan B. Desai, "Comparative analysis of different routing protocols in Delay Tolerant Network," International Journal of Computer Science and Engineering Technology (IJCSET), vol. 4, no. 3, pp. 249-253, 2013.

[2] Forrest Warthman, "Delay and Disruption tolerant Networks (DTNs) A Tutorial," 7 July 2012. [Online].Available: http://www.ipnsig.org. [Accessed 12 September 2016].

[3] Wikipedia, Available: https://en.wikipedia.org/wiki/Routing_in_delay-tolerant_networking. [Accessed 12September 2016]

[4] Chintan B. Desai, "A Survey on Knowlwdge Based Classification of Different Routing Protocols in Delay Tolerant Network," International Journal of Computer Science and Mobile Computing, vol. 2, no. 3, pp. 83-88, 2013 